\title{
Polarized parton densities and spin dependent structure func- tion of the nucleon
}

\author{
M. Salimi-Amiri ${ }^{1, a}$, A. Khorramian ${ }^{1, b}$, H. Abdolmaleki ${ }^{1, c}$, and A. Ghaffari-Turan ${ }^{1, d}$ \\ ${ }^{1}$ Faculty of Physics, Semnan University, 35131-19111, Semnan, Iran
}

\begin{abstract}
We study the polarized parton distribution functions (PPDFs) from recent experimental data at the next-to-leading order (NLO) approximation in the fixed-flavor number scheme. In this analysis, we can compare our results with experimental data such as very recent COMPASS data. It would be very worth to study the PPDFs parametrization form when we want to use this new COMPASS data in QCD analysis on polarized proton structure and parton distribution functions.
\end{abstract}

\section{Introduction}

Determination of fundamental spin structure in terms of their polarized quarks and gluons is one of the main challenges in polarized hadron physics. Despite the spin physics theory of polarized hadrons has been compatible with the experimental data of polarized nucleon, some fundamental aspects of the polarized parton distribution and structure function are still weakly determined. One of the important points in polarized hadron structure is understanding of nucleon's total angular momentum in terms of the single contributions of polarized parton. The total angular momentum of the polarized nucleon in terms of its parton spin, i.e. quark $(\Sigma)$ and gluon $\operatorname{spin}(G)$, and also quark and gluon orbital momentum $\left(L_{q}, L_{g}\right)$ is as following

$$
\frac{1}{2}=\frac{1}{2} \Sigma\left(Q^{2}\right)+G\left(Q^{2}\right)+L_{q}\left(Q^{2}\right)+L_{g}\left(Q^{2}\right),
$$

In this equation, the first two terms present the fraction of the proton spin which carried by quarks and gluons. In fact, it would be worth to be able to measure each contribution in above equation. In this regard, we need to have the polarized parton distribution functions (PPDFs) as a function of $x$ and $Q^{2}$. Also the dependence of the PPDFs on the $Q^{2}$ can be obtained using the DGLAP evolution equations [DGLAP] in perturbative QCD. Very recent analysis on polarized splitting functions in Mellin space has been reported at the next-to-next-to-leading order (NNLO) accuracy. Very recent theoretical and experimental developments in spin physics are addressed in Ref. [1].

We have different analysis to determine PPDFs parametrization such as simple polynomials approach, neural networks for PPDFs parametrization and etc such as DSSV [2-4], NNPDF [5, 6] and

\footnotetext{
ae-mail: Maryam.salimi.amiri@semnan.ac.ir

be-mail: Khorramiana@semnan.ac.ir,Khorramiana@theory.ipm.ac.ir

ce-mail: Abdolmalki@semnan.ac.ir

$\mathrm{d}_{\mathrm{e}-\mathrm{mail}}$ : A.Ghafary@semnan.ac.ir
} 
JAM collaboration. On the other hand, we have a different kind of experimental data such as inclusive and semi-inclusive DIS data. COMPASS and JLAB have been reported very recent DIS measurements [7].

In Refs.[8-16] we have extracted PPDFs from different approaches, such as Jacobi polynomials for PPDFs parameterization up to NLO and different available experimental data.

This paper is organized as follows. In Sec. 2 we present the polarized proton structure in MellinMoment space. The functional form for PPDFs is presented in Sec. 3 at the NLO. Finally our results and conclusions are discussed in Sec. 4.

\section{Formalism}

According to Mellin $N$-moment space, the Mellin moment of function $f$ which is depending on momentum fraction $x$ is defined as:

$$
\mathbf{M}[f, N] \equiv \int_{0}^{1} d x x^{N-1} f(x)
$$

In this regard, the polarized nucleon structure function is defined in terms of PPDFs at the NLO as following [17]

$$
\begin{aligned}
\mathbf{M}\left[g_{1}, N\right]= & \frac{1}{2} \sum_{q} e_{q}^{2}\left\{\left[1+\frac{\alpha_{s}}{2 \pi} \Delta C_{q}^{N}\right]\left[\delta q\left(N, Q^{2}\right)+\delta \bar{q}\left(N, Q^{2}\right)\right]\right. \\
& \left.+\frac{\alpha_{s}}{2 \pi} 2 \Delta C_{g}^{N} \delta g\left(N, Q^{2}\right)\right\}
\end{aligned}
$$

In above equation, $\delta q\left(N, Q^{2}\right), \delta \bar{q}\left(N, Q^{2}\right)$ and $\delta g\left(N, Q^{2}\right)$ are moment of polarized parton distribution functions. Also $\alpha_{s}$ is strong coupling constant which can be determined by the following equation at NLO [18]

$$
\begin{aligned}
\frac{1}{a_{s}\left(Q^{2}\right)}= & \frac{1}{a_{s}\left(Q_{0}^{2}\right)}+\beta_{0} \ln \left(\frac{Q^{2}}{Q_{0}^{2}}\right) \\
& -\beta_{1} \ln \left\{\frac{a_{s}\left(Q^{2}\right)\left[1+\beta_{1} a_{s}\left(Q_{0}^{2}\right)\right]}{a_{s}\left(Q_{0}^{2}\right)\left[1+\beta_{1} a_{s}\left(Q^{2}\right)\right]}\right\},
\end{aligned}
$$

where $a_{s}=\frac{\alpha_{s}}{4 \pi}$ and $\alpha_{s}\left(Q_{0}^{2}\right)$ is strong coupling constant at input scale. In this analysis, we fixed $N_{f}=3$ in the perturbative QCD evolution at Fixed-flavor number scheme at NLO with massless partonic flavors $\{u, d, s\}$. Also we fixed $\alpha_{s}\left(Q_{0}^{2}\right)=0.580$ which is related to $\alpha_{s}\left(M_{Z}^{2}\right)=0.118$.

Now by having the moments of polarized structure function, we have the polarized structure function $x g_{1}\left(x, Q^{2}\right)$ in $x$-space using Jacobi polynomials approach:

$$
x g_{1}\left(x, Q^{2}\right)=x^{\beta}(1-x)^{\alpha} \sum_{n=0}^{N_{\max }} \Theta_{n}^{\alpha, \beta}(x) \times \sum_{j=0}^{n} c_{j}^{(n)}(\alpha, \beta) \mathbf{M}\left[x g_{1}, j+2\right],
$$

where $N_{\max }$ is the number of polynomials which we have considered $N_{\max }=9$ and we used $\alpha=3.0$ and $\beta=0.5$ [9-12]. Also, in above equation $\Theta_{n}^{\alpha, \beta}(x)$ is as following 


$$
\Theta_{n}^{\alpha, \beta}(x)=\sum_{j=0}^{n} c_{j}^{(n)}(\alpha, \beta) x^{j}
$$

So by having $\mathbf{M}\left[x g_{1}, N\right]$ at any values of $Q^{2}$ in Eq. 3, one can determine the polarized structure $x g_{1}\left(x, Q^{2}\right)$ using Jacobi polynomials approach according to Eq. 4 . The comparison of different values of $N_{\text {max }}$ in the NLO approximation at $Q^{2}=2.5 \mathrm{GeV}^{2}$ is presented in Fig. 1.

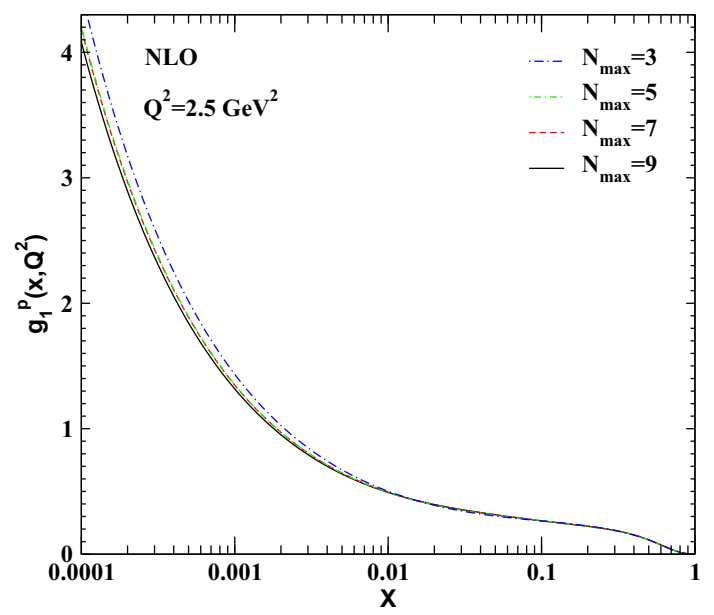

Figure 1. Our results for polarized structure function at $Q^{2}=2.5 \mathrm{GeV}^{2}$ and for different values of $N_{\max }=3,5,7,9$ in Jacobi polynomials approach.

\section{Polarized Parton Distribution}

In this analysis we used the AKS[13] parametrization at $Q_{0}^{2}=1 \mathrm{GeV}^{2}$. According to this parametrization the polarized parton distribution functions ( $\delta q=\delta u+\delta \bar{u}, \delta d+\delta \bar{d}, \delta \bar{u}, \delta \bar{d}, \delta \bar{s}$ and $\delta g$ ) at the NLO and the initial scale are as following:

$$
x \delta q=\mathcal{N}_{q} \eta_{q} x^{a_{q}}(1-x)^{b_{q}}\left(1+c_{q} x^{0.5}+d_{q} x\right)
$$

The normalization constant is $\mathcal{N}_{q}$

$$
\begin{aligned}
\frac{1}{\mathcal{N}_{q}}= & \left(1+d_{q} \frac{a_{q}}{a_{q}+b_{q}+1}\right) B\left(a_{q}, b_{q}+1\right) \\
& +c_{q} B\left(a_{q}+\frac{1}{2}, b_{q}+1\right)
\end{aligned}
$$

and $B(a, b)$ is the Euler beta function. 

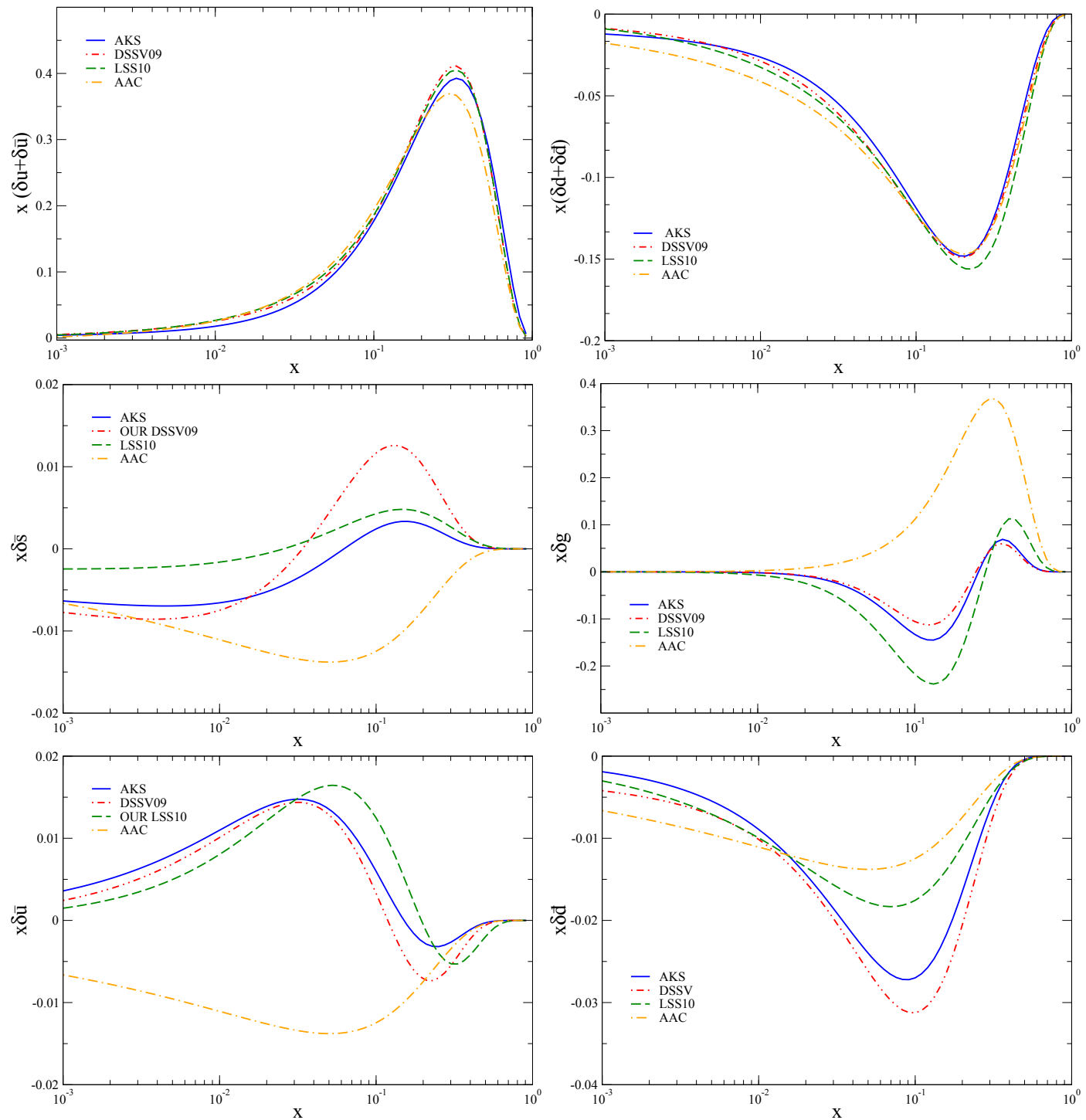

Figure 2. The comparison of parton polarized distribution functions for different parametrization in the NLO approximation, such as DSSV ,LSS, AAC, AKS with an initial scale.

In Fig. 2, we present the evolution of the distribution of polarized PDFs using AKS[13], DSSV[3], LSS[19], AAC[20] parametrization.

In Fig. 3 we show our results for polarized structure function $x g_{1}$ as a function of $x$ at $<Q^{2}>=$ $50 \mathrm{GeV}^{2}$ at the NLO. In this figure, we used the polarized parton distribution functions from Ref. [13] and compare our results with very recent COMPASS experimental data. 


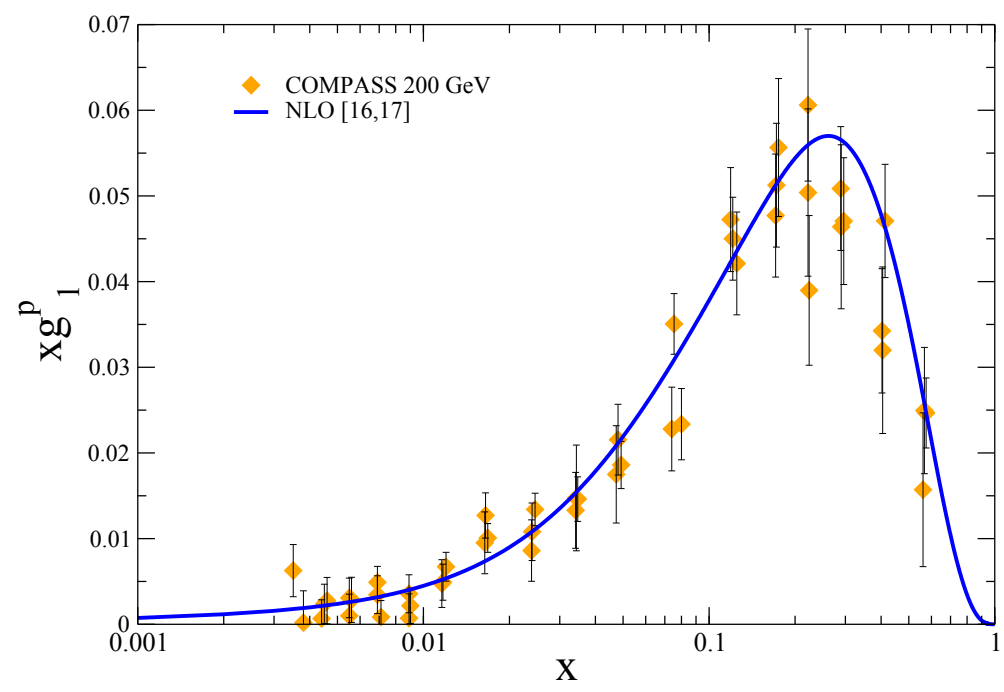

Figure 3. Comparison of polarized structure function $x g_{1}$ with recent experimental COMPASS data at NLO approximation using the polarized parton distribution functions from Ref. [13].

\section{Conclusion}

In this analysis, we investigate the polarized parton distributions at the NLO to see how much compatible our previous parametrization with very recent experimental COMPASS data. Also, we found that the behavior of our parametrization is good agreement with very recent COMPASS data. Our analysis show us the Jacobi polynomial approach is very good approach for extracting the PPDFs in QCD analysis as well.

\section{References}

[1] E. Nocera and S. Pisano, arXiv:1608.08575 [hep-ph].

[2] D. de Florian, R. Sassot, M. Stratmann and W. Vogelsang, Phys. Rev. Lett. 101, 072001 (2008)

[3] D. de Florian, R. Sassot, M. Stratmann and W. Vogelsang, Phys. Rev. D 80, 034030 (2009)

[4] A. Bazavov et al., Phys. Rev. Lett. 113, no. 7, 072001 (2014)

[5] R. D. Ball et al. [NNPDF Collaboration], Nucl. Phys. B 874, 36 (2013)

[6] E. R. Nocera et al. [NNPDF Collaboration], Nucl. Phys. B 887, 276 (2014)

[7] C. Adolph et al. [COMPASS Collaboration], Phys. Lett. B 753, 18 (2016)

[8] S. Taheri Monfared, Z. Haddadi and A. N. Khorramian, Phys. Rev. D 89, no. 7, 074052 (2014)

Erratum: [Phys. Rev. D 89, no. 11, 119901 (2014)]

[9] A. N. Khorramian, S. Atashbar Tehrani and A. Mirjalili, Nucl. Phys. Proc. Suppl. 152, 83 (2006).

[10] S. Atashbar Tehrani and A. N. Khorramian, JHEP 0707, 048 (2007)

[11] A. N. Khorramian, S. Atashbar Tehrani, S. Taheri Monfared, F. Arbabifar and F. I. Olness, Phys. Rev. D 83, 054017 (2011)

[12] A. N. Khorramian and S. Atashbar Tehrani, AIP Conf. Proc. 915, 420 (2007).

[13] F. Arbabifar, A. N. Khorramian and M. Soleymaninia, Phys. Rev. D 89, no. 3, 034006 (2014) 
[14] F. Arbabifar, A. N. Khorramian and M. Soleymaninia, Acta Phys. Polon. Supp. 7, no. 3, 585 (2014).

[15] F. Arbabifar, A. N. Khorramian, H. Khanpour and S. A. Tehrani, Nucl. Phys. Proc. Suppl. 234, 66 (2013)

[16] A. N. Khorramian, S. Atashbar Tehrani, F. Olness, S. Taheri Monfared and F. Arbabifar, Nucl. Phys. Proc. Suppl. 207-208, 65 (2010).

[17] B. Lampe and E. Reya, Phys. Rept. 332, 1 (2000)

[18] A. Vogt, Comput. Phys. Commun. 170, 65 (2005)

[19] E. Leader, A. V. Sidorov and D. B. Stamenov, Phys. Rev. D 82, 114018 (2010) doi:10.1103/PhysRevD.82.114018 [arXiv:1010.0574 [hep-ph]].

[20] Y. Goto et al. [Asymmetry Analysis Collaboration], Phys. Rev. D 62, 034017 (2000) doi:10.1103/PhysRevD.62.034017 [hep-ph/0001046]. 